\title{
Study on Temperature Effect in DAMPE BGO ECAL
}

\author{
Yifeng. Wei \\ State Key Laboratory of Particle Detection and Electronics, Department of Modern Physics, University \\ of Science and Technology of China \\ No. 96, Jinzhai Road, Hefei, China \\ E-mail: weiyfemail.ustc.edu.cn
}

\section{Yunlong. Zhang*}

State Key Laboratory of Particle Detection and Electronics, Department of Modern Physics, University of Science and Technology of China

No. 96, Jinzhai Road, Hefei, China

E-mail: ylzhang@ustc.edu.cn

\section{Zhiyong. Zhang}

State Key Laboratory of Particle Detection and Electronics, Department of Modern Physics, University of Science and Technology of China

No. 96, Jinzhai Road, Hefei, China

E-mail: zhzhy@mail.ustc.edu.cn

\section{Chi. Wang}

State Key Laboratory of Particle Detection and Electronics, Department of Modern Physics, University of Science and Technology of China

No. 96, Jinzhai Road, Hefei, China

E-mail: chiwang@mail.ustc.edu.cn

Abstract-The DArk Matter Particle Explorer (DAMPE) is an orbital experiment which aims at searching dark matter by measuring the spectra of gamma, electron and positron originating from space. The BGO electromagnetic calorimeter (ECAL) is one of the core sub-detectors of DAMPE for energy measurement from $5 \mathrm{GeV}$ to $10 \mathrm{TeV}$. The Calorimeter consists of $308 \mathrm{BGO}$ crystal bars with the dimension of $2.5 \mathrm{~cm} \times 2.5 \mathrm{~cm} \times 60 \mathrm{~cm}$ each. The light optput of BGO crystal depends not only on the energy deposited by particles but also on the temperature. We have studied the temperature dependence of the BGO calorimeter reposonse to cosmic rays in the thermal vacuum chamber. A temperature correction method is also reported in this paper

The 34th International Cosmic Ray Conference

30 July- 6 August, 2015

The Hague, The Netherland 


\section{Introduction}

DArk Matter Particle Explorer (DAMPE) is an orbital experiment aiming at searching for dark matter particle indirectly by measuring the spectra of photons, electrons and positrons originating from deep space. The DAMPE detector is designed to cover a wide energy range, from 5 $\mathrm{GeV}$ to $10 \mathrm{TeV}$ [1], with a good energy resolution of $1.5 \%$ at $800 \mathrm{GeV}$ for photons and electrons, and will be one of the most precise detectors for energy measurement of these particles in an energy range from sub-TeV to tens-TeV. DAMPE will orbit the earth at an altitude of $500 \mathrm{~km}$, it designed to withstand a total dose of $20 \mathrm{k}$ rad during a 3 year mission.

The BGO electromagnetic calorimeter is one of the key sub-detectors of DAMPE. The light output of BGO (Bismuth Germanate) like that of most inorganic scintillation crystal exhibits some dependence on its operating temperature. Since temperature on orbit vary from $-15{ }^{\circ} \mathrm{C}$ to $+20{ }^{\circ} \mathrm{C}$, temperature calibration of BGO calorimeter is necessary. We have investigated the temperature dependence of light yield for single BGO crystal bar [2]. Thermal performance of BGO crystal in full calorimeter is reported in this paper.

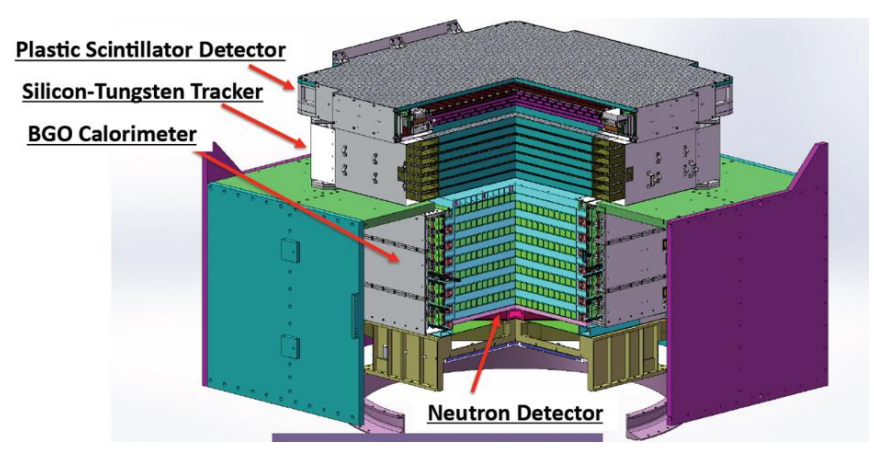

Fig. 1. Sub-detectors of DAMPE

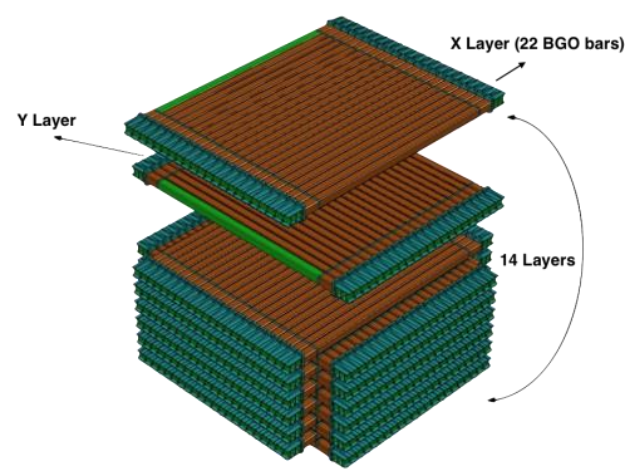

Fig. 2. Structure of DAMPE calorimeter

\section{Calorimeter Module Design}

The DAMPE calorimeter is made up of 308 BGO crystal bars, each with dimensions of $25 \times 25 \times 600 \mathrm{~mm}^{3}$ arranged in 14 layers of 22 crystals ( $~ 31.25$ radiation lengths, 1.6 nuclear interaction length), as shown in Fig. 2. The crystals are supported by a carbon fiber structure. The layers of BGO bars are alternated in an orthogonal way to measure the deposited energy and shape of hadron and electromagnetic showers developed in the crystals.

Scintillation light is detected at each end of a crystal bar by two PMTs respectively. The required range of energy response for one BGO detector element varies from $10 \mathrm{MeV}$ to $2 \mathrm{TeV}$. To obtain a wide dynamic range, a multi-dynode readout structure of PMT base is used [3].

\section{Experiment Procedure}

The engineering qualified module is a full scale prototype built with the same technology as flight modules to verify the design and technological choices. To measure BGO calorimeter performance of temperature in space, a thermal vacuum experiment of DAMPE qualified module was carried out at Shanghai Institute of Satellite Engineering in June, 2014. The BGO calorimeter is hold in a thermal vacuum chamber. The heater plates were pasted on the surface of calorimeter 


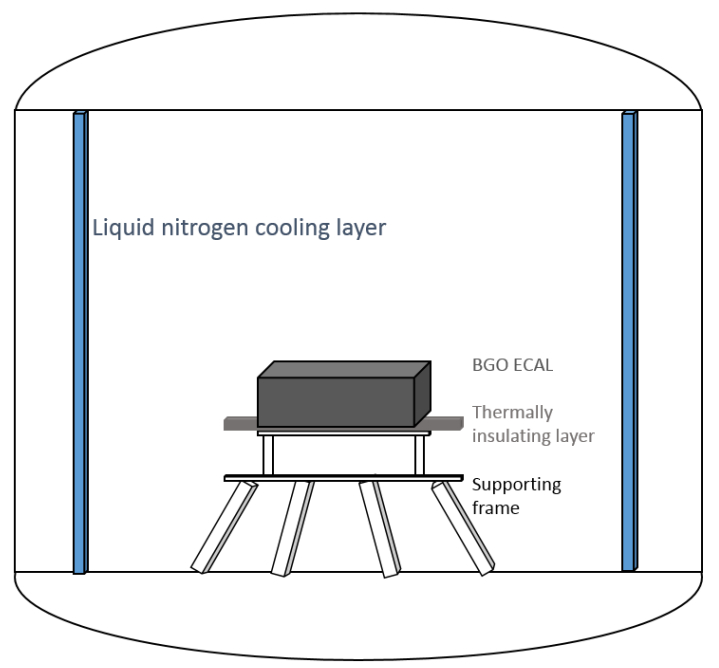

Fig. 3. The BGO calorimeter is put in a thermal vacuum chamber

and the liquid nitrogen cooling layer was around the instrument inside the chamber. The temperature of experiment varied from $-5{ }^{\circ} \mathrm{C}$ to $+30{ }^{\circ} \mathrm{C}$.

\section{Temperature Calibration Result}

Cosmic ray muons was used in thermal vacuum experiment to study the temperature dependence of BGO calorimeter. The cosmic ray data were collected for a duration of about 2 hours, and its spectrum can be fitted with convolution of a Landau with a Gaussian, whose Landau MPV value corresponding to scintillation light output of BGO crystal. Temperature of BGO crystal was readout by thermistor attached on the surface of BGO bar inside of the calorimeter. For each crystal bar, the MPV value decrease with the increase of temperature (Fig. 4), a line was fitted to describe the variation trend of MPV value in this temperature range, as shown in Fig. 5. As an illustration of variation from crystal to crystal Fig. 6 shows the slopes of the BGO crystal bars normalized to $0{ }^{\circ} \mathrm{C}$. The mean value of it is about $-1.2 \%$ per degree Celsius, which contains the contribution of the BGO crystal as well as that of the readout electronics (PMT, base, FEE). A temperature correction algorithm has also been developed using temperature coefficient calibrated in thermal vacuum experiment.

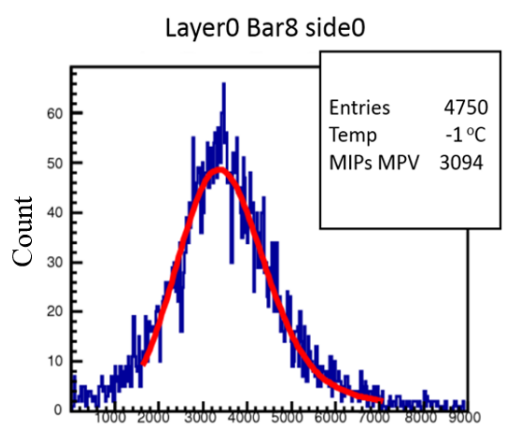

$\mathrm{ADC}$ count

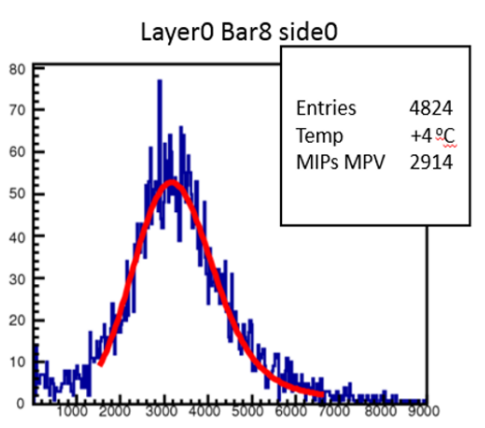

ADC count

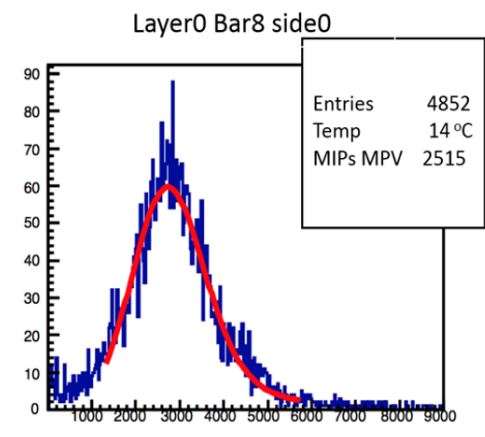

ADC count

Fig. 4. Muon spectrum of individual channel in various temperature 


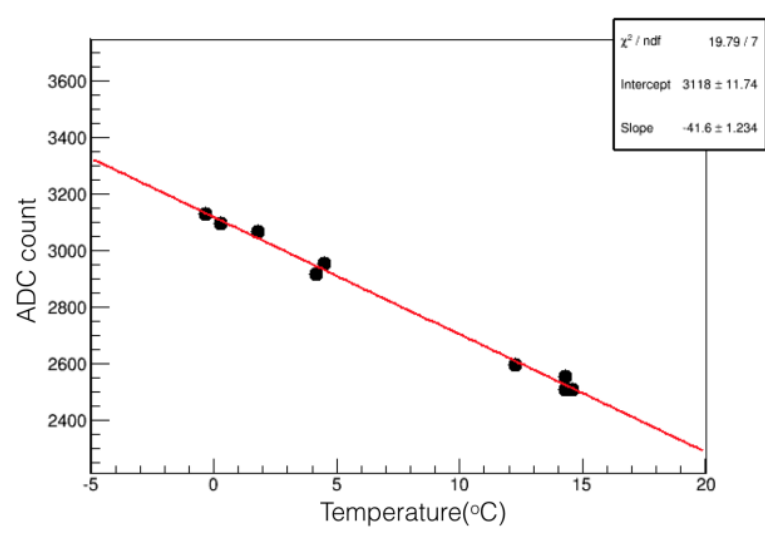

Fig. 5. Temperature dependence of one BGO crystal bar

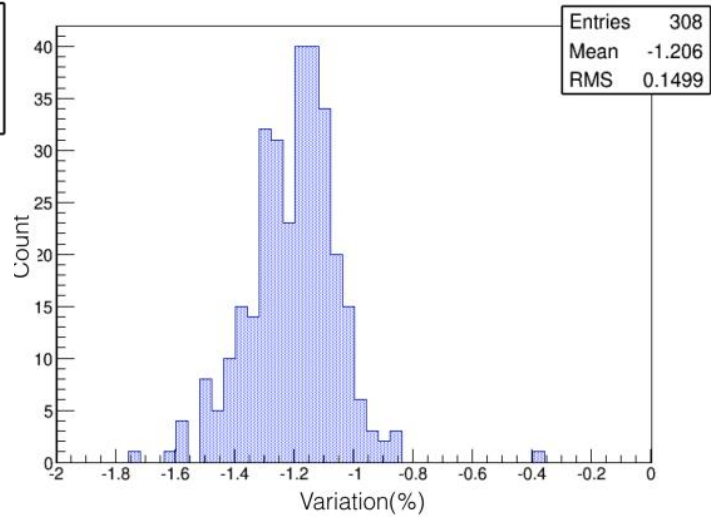

Fig. 6. Distribution of temperature dependance of individual crystal

\section{Conclusion}

The temperature sensitivity of DAMPE BGO calorimeter has been calibrated by utilizing a thermal vacuum chamber at Shanghai Institute of Satellite Engineering. We have confirmed temperature dependence of the light yield of BGO crystal, mean value of temperature coefficient at 0 ${ }^{\circ} \mathrm{C}$ is $-1.2 \%$.

\section{Acknowledgement}

This work was supported by the National Basic Research Program of China, the Strategic Priority Research Program on Space Science of the Chinese Academy of Science.

\section{References}

[1] Y.L. Zhang, B. Li, C.Q. Feng, X.L. Li, X.L. Wang, Z.Z. Xu, et al., "A high dynamic range readout unit for a calorimeter," Chinese Physics C, vol. 36, pp. 71-73, 2012.

[2] P.L. Wang, Y.L. Zhang, Z.Z. Xu, and X.L. Wang, "Study on the temperature dependence of BGO light yield," Science China: Physics, Mechanics and Astronomy, vol. 57, pp. 1898-1901, 2014.

[3] Z.Z. Zhang, Y.L. Zhang, J.N. Dong, S.C. Wen, C.Q. Feng, C. Wang, et al., "Design of a high dynamic range photomultiplier base board for the BGO ECAL of DAMPE," Nuclear Instruments and Methods in Physics Research Section A: Accelerators, Spectrometers, Detectors and Associated Equipment, vol. 780, pp. 21-26, 4/21/ 2015. 